\title{
LAS PERSONAS CON DISCAPACIDAD INTELECTUAL COMO VÍCTIMAS DE DELITOS CONTRA LA LIBERTAD SEXUAL: UNA REALIDAD INVISIBLE
}

\section{People with Intellectual Disability as Victims of Sexual Offences: An Invisible Reality}

\author{
Aránzazu Vellaz Zamorano \\ Universidad de Salamanca \\ Patricia Navas Macho \\ Universidad de Salamanca. Instituto Universitario de Integración en la Comunidad. Departa- \\ mento de Personalidad, Evaluación y Tratamiento Psicológicos \\ patricianavas@usal.es
}

Inés De Araoz SÁNChez-Dopico

Plena inclusión España

Recepción: 21 de abril de 2020

Aceptación: 22 de julio de 2020

RESUMEN: El presente trabajo tiene por objeto conocer los ajustes de procedimiento que se desarrollan durante los procesos judiciales ante hechos constitutivos de abuso o agresión sexual cuando la víctima presenta una discapacidad intelectual o del desarrollo (DID). Además, analiza las características de víctimas con DID y agresores, aportando información adicional sobre el proceso de denuncia. Para ello, se analizaron un total de 56 sentencias resueltas en un periodo de cuatro años (2014-2018), extrayendo información objetiva de las mismas con respecto a los aspectos más relevantes referidos a la víctima, el delito, el agresor y al proceso judicial. Del análisis realizado podemos concluir la existencia de multitud de obstáculos que aún siguen presentes en la práctica judicial española, siendo necesaria una formación exhaustiva de las personas vinculadas a los sistemas procesales en aspectos referidos a esta discapacidad: la víctima declara en reiteradas ocasiones, especialmente cuando se juzga un delito de agresión sexual, sin ningún tipo de adaptación o apoyo profesional. Señalamos asimismo la necesidad de evaluar la 
huella psicológica que pudiera presentar la víctima, así como desterrar mitos con respecto a las discapacidades del desarrollo que dificultan el derecho a acceder y participar en los procedimientos judiciales en condiciones de igualdad.

Palabras Clave: discapacidad intelectual; abuso sexual; agresión sexual; ajustes de procedimiento; victimización.

Aвstract: The following paper aims to analyze the provision of procedural accommodations during the legal process to victims of sexual abuse or sexual assault with an intellectual or developmental disability (IDD). It also examines the characteristics of victims with IDD and their aggressors, providing additional information on the reporting process. To this end, a total of 56 sentences resolved over a period of four years (2014-2018) were analyzed, extracting objective information regarding the most relevant aspects of the crime committed, the victim, the aggressor, and the legal process. The obtained results showed the existence of different obstacles that are still present in the Spanish jurisprudence, requiring an appropriate training for those working in the field of administration of justice: the victim declares repeatedly, especially when a crime of sexual assault is being judged, and without any type of accommodation or professional support. We also point out the need to evaluate the psychological consequences that the victim may experience, and to dispel myths regarding developmental disabilities that hinder access and participation in legal proceedings under equal conditions.

KEYWORDs: intellectual disability; sexual abuse; sexual assault; procedural accommodations; victimization.

T

NO DE los MAYORES AVANCES en la defensa de los derechos de las personas con discapacidad a nivel mundial vino de la mano de la Convención sobre los Derechos de las Personas con Discapacidad (Naciones Unidas, 2006), en vigor en España desde el 3 de mayo de 2008 (en adelante, la Convención). Desde su firma y ratificación, este Tratado Internacional de Derechos Humanos forma parte del ordenamiento jurídico interno, con un rango jerárquico solo por debajo de la Constitución, de cuyos derechos fundamentales es, además, instrumento interpretativo de acuerdo al artículo 10.2 de la misma.

El artículo 16 de la Convención dispone que los Estados Partes adoptarán todas las medidas que sean pertinentes para proteger a las personas con discapacidad contra cualquier forma de explotación, violencia y abuso (Naciones Unidas, 2006, pp. 1314). Además, tal y como detalla en su artículo 13, los Estados Partes asegurarán que las personas con discapacidad tengan "acceso a la justicia en igualdad de condiciones con las demás, incluso mediante ajustes de procedimiento y adecuados a la edad, promoviendo, asimismo, la capacitación adecuada de los que trabajan en la administración de justicia” (Naciones Unidas, 2006, p. 12).

No obstante, como señalan Navas, Gómez, Verdugo y Schalock (2012), nuestra capacidad para implementar sin fisuras el articulado de la Convención está directamente 
relacionada con el grado en que todas las esferas de nuestra sociedad se adhieren al mismo. En este sentido, y como ya denunciara Plena inclusión en los años 2014 y 2018, las personas con discapacidades intelectuales y del desarrollo (DID) siguen haciendo frente a situaciones de vulneración de derechos que se agravan por las dificultades existentes en el acceso a la justicia (De Araoz, 2018; Recover y De Araoz, 2014). Esta situación, como se expondrá a lo largo de este trabajo, parece ser especialmente alarmante cuanto se trata de actos que atentan contra la libertad sexual de las personas con DID (Byrne, 2017; Nixon, Thomas, Daffern y Ogloff, 2017; Wissink, van Vugt, Smits, Moonen y Stams, 2017).

\section{Delitos contra la libertad sexual y personas con discapacidad intelectual: algunos datos}

Nuestro Código Penal (CP) tipifica distintos tipos de delitos contra la libertad e indemnidad sexual, diferenciando entre: abuso, agresión y acoso sexual, abusos y agresiones sexuales a menores de 16 años, exhibicionismo y provocación sexual, prostitución y explotación sexual, y corrupción sexual de menores. Los distintos informes elaborados en nuestro país por el Ministerio del Interior con respecto a esta tipología delictiva ponen de manifiesto que el abuso y la agresión son los delitos contra la libertad sexual más frecuentes, siendo fundamentalmente femenino (con excepción del abuso sexual a menores) el perfil de la víctima (Cereceda et al., 2017, 2018).

La diferencia entre ambos tipos penales (i. e., abuso y agresión sexual) reside, según matiza la Sentencia del Tribunal Supremo 73/2004 de 26 de enero (p. 3), en lo siguiente:

En que, en el primero, el atentado contra la libertad sexual de la víctima se comete viciando el sujeto activo el consentimiento de la misma mediante el prevalimiento de una situación de superioridad, o desconociendo sencillamente la incapacidad de aquella víctima para prestar su consentimiento libre, en tanto en el segundo el atentado se consigue venciendo, mediante la fuerza o la intimidación, la voluntad contraria de la víctima.

Cuando alguno de estos delitos se comete contra víctimas especialmente vulnerables o personas con discapacidad necesitadas de especial protección, el CP otorga una protección reforzada a través de la utilización de agravantes y penas accesorias.

A pesar de la especial vulnerabilidad de las personas con DID contemplada en nuestro $\mathrm{CP}$, encontrar datos precisos sobre el grado en que son víctimas de este tipo de delitos resulta una tarea casi imposible en nuestro país por diversos motivos. En primer lugar, la libertad sexual de las personas con DID no ha sido objeto de interés hasta hace unas dos décadas (Byrne, 2017). En segundo lugar, las personas con discapacidades del desarrollo pueden encontrar especiales dificultades de acceso a la información que obstaculicen el proceso de denuncia, por lo que los datos disponibles solo representan una parte del conjunto de los delitos realmente cometidos (De Araoz, 2018; Nixon et al., 2017; Verdugo, Alcedo y Aguado, 2002). En tercer lugar, los datos 
oficiales sobre victimización, como los ofrecidos por el Ministerio del Interior, no realizan mención alguna a la presencia de discapacidad. Por último, son escasos los estudios que analizan de manera particular la vulnerabilidad de las personas con DID frente a esta tipología delictiva sin incluir otro tipo de situaciones de maltrato o abuso o hacer alusión a otras víctimas necesitadas de especial protección como los menores de edad (Byrne, 2017).

Superando estas limitaciones, algunos estudios recientes arrojan datos que alertan sobre la especial vulnerabilidad que, frente a la población general, experimentan aquellas personas con DID en relación con los delitos contra la libertad sexual más frecuentes: abuso y agresión. Así, Nixon et al. (2017) estiman que presentan un riesgo casi seis veces mayor al observado en la población general. Es, además, más probable que experimenten reiterados episodios de victimización (Fisher, Baird, Currey y Hodapp, 2016; Nixon et al., 2017). Resultados similares han sido arrojados por Spencer et al. (2005) y Withers y Morris (2012) al estudiar de manera específica el delito de abuso sexual a menores con DID, estimando un riesgo entre seis y cuatro veces mayor con respecto a sus iguales sin discapacidad, respectivamente.

Entre los factores que explican esta mayor vulnerabilidad, la literatura científica señala: (a) las mayores dificultades para identificar y enfrentarse a situaciones de riesgo (Fisher et al., 2016; Greenspan y Woods, 2014; Helton, Gochez-Kerr y Gruber, 2018; Mevissen y De Jongh, 2010); (b) el ser percibidos por el abusador o agresor como personas "más fáciles de engañar” y "menos creíbles" (Beail, 2002; Byrne, 2017); (c) la frecuente dependencia de terceros, que les resta intimidad para la realización de actividades de la vida cotidiana (Byrne, 2017; Recio, Alemany y Manzanero, 2012; Recio et al., 2013; Wissink et al., 2017); (d) la sobreprotección del entorno, que se traduce en que no preparemos a las personas con DID para protegerse ante este tipo de delitos al querer evitar que los conozcan (Byrne, 2017; Fisher et al., 2016; Recio et al., 2012; Recio et al., 2013), entre otros. A estos factores hemos de sumar actitudes y creencias basadas en mitos y prejuicios sobre la sexualidad de las personas con DID (analizadas en profundidad por Verdugo et al., 2002) que limitan aún más el acceso a una educación sexual apropiada e incrementan el riesgo frente a situaciones de abuso.

\section{Una mayor vulnerabilidad que se acompaña de barreras en el acceso a la justicia}

Las personas con DID no solo son más vulnerables frente al abuso o agresión sexual, sino que, además, se encuentran con especiales dificultades para navegar por el complejo sistema de justicia, tanto a la hora de interponer una denuncia como participando en calidad de testigos en el proceso judicial (De Araoz, 2018; Benedet y Grant, 2007; Recio et al., 2013). La existencia de barreras en el acceso a la justicia se traduce en distintas consecuencias para las personas con DID. Una de ellas hace referencia al escaso porcentaje de denuncias y su posterior tramitación. En Reino Unido, de las 1.400 denuncias de abuso sexual a personas con DID que son interpuestas anualmente, solo el 6 \% llega a juicio (Byrne, 2017). En nuestro país, Manzanero, Recio, Alemany y Cendra (2013) señalan que las personas con DID tienden a ser excluidas 
por los sistemas judiciales al considerar que pueden ver mermada su capacidad para elaborar relatos complejos, lo que sin duda puede disminuir la probabilidad de que aquellos que sufren este tipo de delitos denuncien, al vislumbrar un escenario en el que pueden no ser creídos. Esto nos lleva a querer profundizar en este trabajo en una de las mayores dificultades que presenta nuestro sistema de justicia en relación con el tratamiento de víctimas con DID: la revictimización o victimización secundaria.

La victimización secundaria puede definirse como una forma de violencia institucional derivada de la inadecuada atención que recibe la víctima una vez entra en contacto con el sistema judicial (Beristain, 1994). Esta victimización secundaria puede producirse asimismo a nivel social (e. g., mediante la atribución de responsabilidad a la víctima), si bien nos centraremos en aquella que se produce en un sistema que debiera proteger a quien denuncia.

Las personas con DID pueden verse expuestas a diversas situaciones que incrementan las posibilidades de victimización secundaria en el contexto judicial. En primer lugar, y a pesar de que contamos con revisiones extensas que ponen de manifiesto la capacidad de las víctimas con DID para proporcionar información certera sobre hechos que han presenciado (e. g., Beail, 2002; Hershkowitz, 2018; Kebbell y Hatton, 1999), en el contexto judicial suelen ser vistas como testigos poco creíbles (Beail, 2002). Esta tendencia a no otorgar credibilidad al testimonio de la víctima con DID puede verse exacerbada si la persona presenta dificultades comprensivas y/o comunicativas (Greenspan, Switzky y Woods, 2011; Recio et al., 2013). Si bien es cierto que las personas con DID pueden presentar dificultades en los sistemas de acceso y recuperación de la información (e. g., Ford et al., 2017; Palomino, López-Frutos, Botella y Sotillo, 2019), estas dificultades no deben confundirse con una alteración u omisión voluntaria del testimonio. En segundo lugar, las personas con DID pueden ser excluidas del proceso al ser etiquetadas como "fácilmente sugestionables". Como señalan Subijana y Echeburúa (2018), la huella mnésica resulta "fácil de contaminar en víctimas vulnerables por razón de su edad, capacidad intelectual o equilibrio emocional" (p. 22). No obstante, esta manipulación parece estar más relacionada con la naturaleza de las preguntas formuladas (i. e., sugestivas en lugar de abiertas) y con las técnicas de interrogación utilizadas que con la mera presencia de una discapacidad del desarrollo (Beail, 2002; Brown, Lewis, Stephens y Lamb, 2017; Griego, Datzman, Estrada y Middlebrook, 2019; Hershkowitz, 2018; Perske, 2008; Recio et al., 2012). En tercer y último lugar, el conjunto de falacias sobre la sexualidad de las personas con DID puede promover prejuicios entre los profesionales que deben valorar las consecuencias del abuso o agresión o juzgar la responsabilidad jurídico-penal de quien cometió el delito, con consecuencias perjudiciales para la víctima (Verdugo et al., 2002).

3. Ajustes de procedimiento para garantizar el adecuado acceso a la justicia y protección de las personas con DID víctimas de delitos contra la libertad sexual

Una de las herramientas de las que disponemos para prevenir esta victimización secundaria, así como para garantizar la adecuada protección de las personas con DID 
que acceden al sistema de justicia al haber sufrido un delito contra su libertad sexual, consiste en la puesta en marcha de ajustes de procedimiento. Los ajustes de procedimiento son "un medio para hacer valer efectivamente el derecho a un juicio imparcial y el derecho a participar en la administración de justicia”, debiendo proporcionarse sobre la base de "la libre elección y las preferencias del interesado", destacando entre aquellos que podemos emplear con personas con DID los siguientes (Oficina del Alto Comisionado de Derechos Humanos, 2017, p. 8): aquellos procedimientos que garanticen la accesibilidad y el acceso a la información (e. g., adaptación de información jurídica a lectura fácil, intérprete de lengua de signos); flexibilidad en los plazos y modificación de los procedimientos y prácticas relativos a las salas de audiencia; la realización de la prueba preconstituida (i. e., grabación de la declaración para evitar la presencia de la víctima durante el acto de juicio oral y la consecuente repetición del testimonio) y anticipada (i. e., declaraciones testificales recogidas con antelación al juicio), o la incorporación de apoyos durante el proceso que faciliten la participación de la víctima y eviten la repetición constante de la declaración en condiciones no óptimas para ella. En referencia a este último aspecto, se ha de hacer mención a la figura del facilitador en la investigación policial y judicial con víctimas con DID que la Unidad de Atención a Víctimas con Discapacidad Intelectual (UAVDI) de la Fundación A LA PAR propone para solventar algunas de las dificultades de acceso a la justicia comentadas (Manzanero et al., 2013; Recio et al., 2012; Recio et al., 2013). El facilitador es una persona autónoma, experta en discapacidad, cuyas principales funciones radican en informar a la víctima con DID sobre el funcionamiento del sistema policial y judicial, ayudarla a decidir si quiere denunciar, evaluar los factores que puedan afectar a la investigación, asesorar a las autoridades sobre las adaptaciones pertinentes que deban realizarse y diseñar los apoyos requeridos para la adecuada obtención del testimonio (Recio et al., 2012, pp. 62-63; Recio et al., 2013, p. 59). Esta figura es esencial asimismo para evitar la posible manipulación del testimonio de la persona con DID, algo que puede suceder con mayor facilidad en este colectivo ante situaciones sociales complejas (Greenspan et al., 2011).

Como puede apreciarse, existen métodos para posibilitar la participación de las personas con DID en el sistema de justicia, maximizando la protección de la víctima. No obstante, tal y como constató en 2019 el Comité de Naciones Unidas sobre los Derechos de las Personas con Discapacidad en su informe sobre el cumplimiento de la Convención en España, estos métodos rara vez son tomados en consideración por la doctrina.

Por los motivos expuestos, el objetivo principal del presente trabajo radica en analizar, a partir del estudio de sentencias judiciales, el grado en que se ponen en marcha ajustes de procedimiento durante la fase procesal cuando nuestro sistema de justicia se encuentra con víctimas con DID que alegan haber sufrido delitos contra su libertad sexual.

Adicionalmente, se analizarán las características de las víctimas con DID, así como las de sus agresores y el delito cometido, observando, además, si entre los profesionales del sistema judicial aparecen mitos y sesgos con respecto a las personas con DID y su sexualidad. 


\section{Metodología}

El trabajo aquí presentado ha sido elaborado a partir del análisis descriptivo de un conjunto de sentencias de delitos contra la libertad e indemnidad sexual de personas con DID extraídas de la base de datos del ámbito del Derecho, Aranzadi, a finales del año 2018. Los términos de búsqueda utilizados fueron "discapacidad y abuso sexual" y, posteriormente, “discapacidad y agresión sexual”. La búsqueda se limitó a los últimos cuatro años (2014-2018) con el objetivo de tener en cuenta la ratificación por parte de España en 2014 del Convenio de Estambul sobre prevención y lucha contra la violencia contra la mujer y la violencia doméstica, así como los cambios acontecidos tras la entrada en vigor de la Ley 4/2015, de 27 de abril, del Estatuto de la víctima del delito. Ambos documentos recogen distintas medidas para proteger a la víctima durante el proceso judicial (e. g., toma de declaración por parte de expertos y haciendo uso de tecnologías de la comunicación e información).

De la búsqueda efectuada resultaron un total de 46 sentencias de abuso y 35 de agresión sexual $(\mathrm{N}=81)$. Un total de 25 sentencias fueron excluidas del análisis por los siguientes motivos: (a) referirse a víctimas con otro tipo de discapacidad o indicar exclusivamente el porcentaje de discapacidad de la víctima, no siendo posible conocer la etiología de la misma ( $n=22)$; (b) la sentencia fue anulada o casada, no pudiendo acceder a su resolución definitiva en el momento de la búsqueda $(n=2)$; y (c) no referirse a un delito contra la libertad sexual $(n=1)$. Se analizaron finalmente un total de 56 sentencias, 36 referentes a delitos de abuso sexual y 20 sobre agresión sexual.

Tras la lectura de las sentencias por parte de la primera autora, se identificaron los aspectos más relevantes de cada una de ellas, referidos a la víctima, la identificación del delito y el agresor, así como al proceso judicial. Ante la existencia de dudas en el análisis de estos aspectos, la primera y la segunda autora revisaron la sentencia de manera conjunta para alcanzar un acuerdo. Se recogió, además, información sobre el empleo de terminología apropiada para hacer referencia a la discapacidad de la víctima. Las variables objeto de análisis se detallan en la Tabla 1.

\begin{tabular}{|l|l|l|l|}
\hline \multicolumn{3}{|c|}{ Tabla 1. Aspectos analizados en las sentencias objeto de estudio } \\
\hline Víctima & Delito & Agresor & Proceso judicial \\
\hline $\begin{array}{l}\text { Grado y tipo de } \\
\text { discapacidad }\end{array}$ & $\begin{array}{l}\text { Persona que inter- } \\
\text { pone la denuncia }\end{array}$ & Relación con la víctima & $\begin{array}{l}\text { Prueba de cargo (i. } \\
\text { e., la que acredita } \\
\text { la existencia del de- } \\
\text { lito y/o la respon- } \\
\text { sabilidad penal del } \\
\text { procesado) }\end{array}$ \\
\hline $\begin{array}{l}\text { Otras discapaci- } \\
\text { dades presentes }\end{array}$ & $\begin{array}{l}\text { Tiempo transcu- } \\
\text { rido entre denun- } \\
\text { cia y sentencia }\end{array}$ & Antecedentes penales & $\begin{array}{l}\text { Pruebas realizadas } \\
\text { tales como: testifi- } \\
\text { cales, psicológicas, } \\
\text { médicas u otras }\end{array}$ \\
\hline
\end{tabular}




\begin{tabular}{|l|l|l|l|}
\hline Sexo & Abuso o agresión & Reincidencia & $\begin{array}{l}\text { Número de decla- } \\
\text { raciones tomadas a } \\
\text { la víctima }\end{array}$ \\
\hline Edad & $\begin{array}{l}\text { Agresión o abuso } \\
\text { puntual o de ca- } \\
\text { rácter continuado }\end{array}$ & $\begin{array}{l}\text { Adaptaciones para } \\
\text { la obtención del tes- } \\
\text { timonio (e. g., el tipo } \\
\text { de preguntas reali- } \\
\text { zadas, espacios em- } \\
\text { pleados para la toma } \\
\text { de declaración) }\end{array}$ \\
\hline & Edad & $\begin{array}{l}\text { Apoyos en la testi- } \\
\text { ficación ofrecidos } \\
\text { por expertos a las } \\
\text { personas con DID } \\
\text { Utilización de la } \\
\text { prueba anticipada o } \\
\text { preconstituida } \\
\text { Valoración de la } \\
\text { huella psíquica o } \\
\text { daño psicológico } \\
\text { Adaptación } \\
\text { terminológica }\end{array}$ \\
\hline
\end{tabular}

\section{Resultados}

Antes de proceder a mostrar los resultados agregados derivados del análisis descriptivo de las diferentes sentencias, se ha de puntualizar que, del total de sentencias estudiadas $(\mathrm{N}=56)$, el número de sujetos asciende a un total de 43 víctimas de abuso sexual (en las 36 sentencias analizadas encontramos cinco situaciones de abuso de dos víctimas con DID y un delito con tres víctimas) y 24 de agresión sexual (en un caso son múltiples las víctimas del delito). Por lo tanto, los datos referidos a las víctimas harán referencia al total de personas con DID que se han visto inmersas en los casos objeto de estudio $(\mathrm{N}=67)$. Asimismo, los datos referidos a las pruebas realizadas superarán en número al total de sentencias revisadas e incluso al número de víctimas, pues una persona puede ser objeto de más de un tipo de prueba (e. g., psicológica y médica). Por otra parte, los datos referidos a las personas que cometen el abuso sexual, a pesar de haber analizado 36 sentencias referidas a esta tipología delictiva, ascenderán a 37, en tanto que en una ocasión se vieron implicados dos agresores.

\subsection{Características de la víctima}

Ediciones Universidad de Salamanca / CC BY-NC-ND

Siglo Cero, vol. 52 (1), 2021, enero-marzo, pp. 7-26 
El análisis realizado pone de manifiesto que la mayor parte de las víctimas (Tabla 2) presenta una discapacidad superior al $65 \%$ (51,2 \% de los casos en los delitos de abuso sexual y $66,7 \%$ en los de agresión sexual), siendo casi en su totalidad víctimas femeninas ( $86 \%$ y $91,7 \%$, respectivamente). Aproximadamente el $40 \%$ de las víctimas estaban tuteladas.

Estos datos reflejan la doble vulnerabilidad que ante situaciones de agresión o abuso presentan las mujeres con DID, especialmente aquellas cuyas limitaciones en funcionamiento intelectual y conducta adaptativa son más severas. Por otro lado, las víctimas de abusos sexuales son más jóvenes $(M=20,5 ; D T=8,4)$ que las víctimas de agresión $(M=30,4 ; D T=14,9)$, representando los menores de edad el $46,5 \%$ de los casos de abuso (16,7 \% si hablamos de agresiones). No obstante, hemos de tener en cuenta que este dato no está disponible en un considerable número de las sentencias analizadas.

TABLA 2. Características objeto de análisis en las sentencias revisadas referidas a la víctima

\begin{tabular}{|c|c|c|}
\hline & $\begin{array}{l}\text { Abuso sexual* } \\
(\mathrm{N}=43)\end{array}$ & $\begin{array}{l}\text { Agresión sexual* } \\
(\mathrm{N}=\mathbf{2 4})\end{array}$ \\
\hline \multicolumn{3}{|c|}{ Grado de discapacidad } \\
\hline Del 33 al $44 \%$ & 11 & 5 \\
\hline Del 45 al $64 \%$ & 6 & 3 \\
\hline Del 65 al $74 \%$ & 16 & 8 \\
\hline Más de $75 \%$ & 6 & 8 \\
\hline No se menciona & 4 & - \\
\hline \multicolumn{3}{|l|}{ Víctima tutelada } \\
\hline Sí & 17 & 10 \\
\hline No & 26 & 14 \\
\hline \multicolumn{3}{|c|}{ Otras discapacidades presentes } \\
\hline Sí & 2 & 1 \\
\hline No & 41 & 23 \\
\hline \multicolumn{3}{|l|}{ Sexo } \\
\hline Mujer & 37 & 22 \\
\hline Hombre & 6 & 2 \\
\hline \multicolumn{3}{|l|}{ Edad } \\
\hline
\end{tabular}


LAS PERSONAS CON DISCAPACIDAD INTELECTUAL COMO VÍCTIMAS DE DELITOS CONTRA LA LIBERTAD SEXUAL: UNA REALIDAD INVISIBLE

ARÁNZAZU VELLAZ ZAMORANO, PATRICIA NAVAS MACHO E INÉS DE ARAOZ SÁNCHEZ-DOPICO

\begin{tabular}{|l|c|c|}
\hline Menor de 18 años & 20 & 4 \\
\hline De 18 a 26 años & 8 & 3 \\
\hline Más de 27 años & 6 & 5 \\
\hline No se menciona & 9 & 12 \\
\hline
\end{tabular}

* Los datos se refieren al número de víctimas y no al número de sentencias analizadas en cada caso.

\subsection{Características del delito y del agresor}

Con respecto a la identificación del delito (Tabla 3), el 58,3 \% de las situaciones de abuso sexual fueron denunciadas por el núcleo familiar, interponiendo la denuncia la víctima solo en el 19,4 \% de los casos. Si bien no hay información precisa en la mayor parte de las sentencias analizadas con respecto al momento en que se interpone la denuncia, sí podemos afirmar que en el 91,6 \% de los casos transcurre un periodo igual o superior a dos años entre la comisión del delito y la ejecución de la sentencia (en el 47,2 \% de las situaciones este periodo es igual o superior a cuatro años). En un $41,7 \%$ de los casos nos encontramos ante un delito de abuso sexual continuado. El agresor fue considerado culpable del delito de abuso sexual en el 90,7 \% de los hechos enjuiciados.

Estos datos varían para el delito de agresión sexual donde se observa un mayor porcentaje de casos en que la propia víctima interpone la denuncia (30 \%). En el 70 \% de las ocasiones transcurre un periodo igual o superior a dos años entre la comisión del delito y la ejecución de la sentencia. En un 30 \% de los casos observamos un delito de agresión sexual continuado. El agresor fue considerado culpable del delito de abuso sexual en el 66,7 \% de los hechos enjuiciados.

\begin{tabular}{|l|c|c|}
\hline \multicolumn{2}{|c|}{ Tabla 3. Características referidas al delito } \\
\hline & $\begin{array}{c}\text { Abuso sexual* } \\
(\mathbf{N}=36)\end{array}$ & $\begin{array}{c}\text { Agresión sexual * } \\
(\mathbf{N}=20)\end{array}$ \\
\hline Características del delito & & \\
\hline $\begin{array}{l}\text { Persona que interpone } \\
\text { la denuncia }\end{array}$ & & 6 \\
\hline Víctima/s & 7 & 8 \\
\hline Familiar/es & 21 & 2 \\
\hline Profesionales & 2 & 4 \\
\hline Persona desconocida & 1 & - \\
\hline No se menciona & 5 & \\
\hline
\end{tabular}

Ediciones Universidad de Salamanca / CC BY-NC-ND

Siglo Cero, vol. 52 (1), 2021, enero-marzo, pp. 7-26 


\begin{tabular}{|l|c|c|}
\hline $\begin{array}{l}\text { Tiempo transcurrido entre } \\
\text { denuncia y sentencia }\end{array}$ & & \\
\hline 1 año & 1 & 4 \\
\hline 2 años & 9 & 3 \\
\hline 3 años & 7 & 1 \\
\hline 4 años & 3 & 4 \\
\hline 5 o más años & 14 & 2 \\
\hline No se menciona & 2 & 6 \\
\hline $\begin{array}{l}\text { Abuso o agresión de carácter } \\
\text { continuado }\end{array}$ & 15 & 13 \\
\hline Sí & 19 & 1 \\
\hline No & 2 & 8 \\
\hline No se menciona & & 16 \\
\hline Sentencia & 4 & \\
\hline Absolutoria & 39 & \\
\hline Condenatoria & & \\
\hline
\end{tabular}

*Los datos se refieren al delito juzgado, y no al número de víctimas en los mismos, salvo en el caso de la variable "sentencia” donde se ha tenido en cuenta el número de hechos delictivos (igual al número de víctimas: 43 en el caso de abuso y 24 para agresión).

Teniendo en cuenta a la totalidad de personas $(\mathrm{N}=57)$ que perpetran el delito (ya sea abuso o agresión), observamos que el perfil de quien comete el mismo (Tabla 4) se caracteriza por pertenecer al círculo social cercano de la víctima (77,2 \% de los casos), siendo superior el porcentaje de miembros de la familia que cometen delitos de abuso sexual (37,8 \% frente al $15 \%$ en el caso de la agresión sexual). Todas las personas que cometen el delito son varones, y, teniendo en cuenta aquellos casos en los que se dispone de información con respecto a su edad, presentan, en su mayoría $(76,7 \%)$, edades superiores a los 35 años $(M=51,5 ; D T=16,6)$. Los antecedentes delictivos previos al delito sexual estuvieron presentes en el 22,8 \% de los casos, siendo reincidentes en un 7,01\% de las ocasiones. 


\begin{tabular}{|c|c|c|c|}
\hline & $\begin{array}{l}\text { Abuso sexual* } \\
\qquad(N=37)\end{array}$ & $\begin{array}{l}\text { Agresión sexual* } \\
\qquad(\mathrm{N}=20)\end{array}$ & Total \\
\hline \multicolumn{4}{|l|}{ Relación con la víctima } \\
\hline Familiar & 14 & 3 & 17 \\
\hline Conocido (no familia) & 15 & 12 & 27 \\
\hline Desconocido & 8 & 5 & 13 \\
\hline \multicolumn{4}{|l|}{ Sexo del acusado } \\
\hline Mujer & - & - & 0 \\
\hline Hombre & 37 & 20 & 57 \\
\hline \multicolumn{4}{|l|}{ Edad del acusado } \\
\hline Menor de 18 años & - & - & 0 \\
\hline De 18 a 35 años & 8 & 2 & 10 \\
\hline De 36 a 54 años & 10 & 6 & 16 \\
\hline De 54 a +65 & 11 & 6 & 17 \\
\hline $\begin{array}{l}\text { No se menciona la } \\
\text { edad del agresor }\end{array}$ & 8 & 6 & 14 \\
\hline \multicolumn{4}{|c|}{ Antecedentes penales del agresor } \\
\hline Sí & 9 & 4 & 13 \\
\hline No & 28 & 16 & 44 \\
\hline \multicolumn{4}{|l|}{ Reincidencia } \\
\hline Sí & 2 & 2 & 4 \\
\hline No & 35 & 18 & 53 \\
\hline
\end{tabular}

* Los datos se refieren al número de agresores ( $\mathrm{N}=37$ en abuso y $\mathrm{N}=20$ en agresión) y no al número de sentencias analizadas en cada caso.

\subsection{Proceso judicial}

En la Tabla 5 se sintetizan los datos más relevantes observados con respecto a las características del proceso judicial. Pese a que en la mayoría de las sentencias la prueba de cargo recae en la declaración de la víctima, es destacable que la mayor parte de las pruebas que se efectúan durante el proceso son testificales (45,4 \% en el caso de abuso y $46 \%$ en agresión). Esto da lugar a que la víctima declare entre dos y cuatro veces en un 32,6\% de los casos de abuso y en un $75 \%$ de los casos de agresión. Esta 
declaración se efectúa sin ningún tipo de adaptación en el 81,4% de las situaciones de abuso y casi en la práctica totalidad de los casos de agresión sexual. Tampoco se ofrecen apoyos profesionales durante la misma, siendo residuales las ocasiones en que esto sí sucede (7 \% en los casos de abuso y 4,1\% en agresión sexual). La prueba preconstituida o anticipada fue utilizada con el 37,2 \% de las víctimas de abuso, y tan solo con cuatro $(16,6 \%)$ de las 24 víctimas de agresión sexual.

TABLA 5. Características objeto de análisis referidas al proceso

\begin{tabular}{|c|c|c|}
\hline & $\begin{array}{l}\text { Abuso sexual } \\
\left(\mathrm{N}=43 / 36^{*}\right)\end{array}$ & $\begin{array}{c}\text { Agresión sexual } \\
(N=24 / 20 *)\end{array}$ \\
\hline \multicolumn{3}{|l|}{ Prueba de cargo } \\
\hline Testificación de la víctima & 39 & 22 \\
\hline Testificación acusado/testigo & 7 & 1 \\
\hline Otras & 3 & 1 \\
\hline No se menciona & 1 & - \\
\hline \multicolumn{3}{|l|}{ Pruebas realizadas } \\
\hline Testificales & 40 & 23 \\
\hline Psicológicas & 28 & 13 \\
\hline Médicas & 17 & 13 \\
\hline Otras & 1 & - \\
\hline No se menciona & 2 & 1 \\
\hline \multicolumn{3}{|l|}{ N. ${ }^{\circ}$ de declaraciones tomadas } \\
\hline Ninguna & - & 1 \\
\hline Solo 1 & 23 & 4 \\
\hline $\begin{array}{l}\text { Entre } 2 \text { y } 4 \\
\text { No se menciona }\end{array}$ & $\begin{array}{c}14 \\
6\end{array}$ & $\begin{array}{c}18 \\
1\end{array}$ \\
\hline \multicolumn{3}{|c|}{ Adaptaciones en la obtención del testimonio } \\
\hline Sí & 8 & 1 \\
\hline No & 35 & 23 \\
\hline \multicolumn{3}{|l|}{ Apoyos durante la testificación } \\
\hline Sí & 3 & 1 \\
\hline No & 1 & 1 \\
\hline No se menciona & 39 & 22 \\
\hline \multicolumn{3}{|l|}{ Prueba preconstituida } \\
\hline Sí & 16 & 4 \\
\hline
\end{tabular}


LAS PERSONAS CON DISCAPACIDAD INTELECTUAL COMO VÍCTIMAS DE DELITOS CONTRA LA LIBERTAD SEXUAL: UNA REALIDAD INVISIBLE

ARÁNZAZU VELLAZ ZAMORANO, PATRICIA NAVAS MACHO E INÉS DE ARAOZ SÁNCHEZ-DOPICO

\begin{tabular}{|c|c|c|}
\hline No & 27 & 20 \\
\hline \multicolumn{3}{|l|}{ Huella psicológica } \\
\hline Sí & 12 & 6 \\
\hline No & 1 & - \\
\hline No se menciona & 30 & 18 \\
\hline \multicolumn{3}{|c|}{ Adaptación terminológica } \\
\hline Sí & 7 & 6 \\
\hline No & 29 & 14 \\
\hline
\end{tabular}

* En esta tabla los totales se corresponden con el total de personas con DI presentes en las sentencias, a excepción del dato de “adaptación terminológica” cuyo total se corresponde con el número de sentencias analizadas. Nótese, además, que, en lo referido a pruebas realizadas, el total superará al número de personas con DI, al poder realizar más de un tipo de prueba en cada caso.

Otro de los aspectos valorados en las sentencias revisadas que merece la pena destacar es el referido a la valoración de la huella psíquica o daño psicológico en la víctima. Solo en uno de cada cuatro casos, aproximadamente, se valora de manera clara el daño psicológico que el abuso o agresión ha producido en la víctima. En un caso concreto, incluso se decide no hacerlo por argumentos que atentan claramente contra el articulado de la Convención, como el observado en la sentencia 147/2016 de 30 de junio de la Audiencia Provincial de A Coruña. En ella, se alega (p. 6) "inexistencia de huella psíquica” tras el abuso sexual, matizando que "dadas las carencias en las funciones cognitivas, la falta de huella psicológica es lo esperable en estos casos”.

El análisis detenido de las sentencias ha permitido analizar otra serie de consideraciones que, si bien pueden aparecer en casos puntuales, deben alertarnos sobre la necesidad de trabajar posibles estereotipos y prejuicios con respecto a las personas con DID, pues estas concepciones erróneas pudieran afectar a la percepción de credibilidad del testimonio de las víctimas y se extienden a su condición de víctimas y la posibilidad de que puedan ser consideradas objeto de deseo o incluso abuso y agresión sexual. Así, en la sentencia sobre abuso sexual 478/2016 de 2 de junio del Tribunal Supremo de Madrid (p. 7), los requisitos de valoración del testimonio que suelen emplearse cuando el relato de la víctima es la única prueba de cargo (i. e., ausencia de incredibilidad subjetiva, verosimilitud persistente en la incriminación y persistencia en la incriminación) son tomados en consideración solo para el testimonio de la víctima que presenta una discapacidad del desarrollo, no siendo así para la otra víctima sin discapacidad, alegando el Tribunal que no era necesario "por ser una persona adulta normal”. Citando otro ejemplo de los estereotipos mencionados, en la sentencia 35/2016 de 23 de febrero de la Audiencia Provincial de Guipúzcoa (p. 6) el tribunal llega a realizar alusiones al aspecto físico de la víctima con DI, como si, por este último motivo, las circunstancias vividas fueran, de algún modo, susceptibles de ser justificadas. Así, en la prueba testifical de la víctima toman como punto de partida 
lo siguiente: “que debemos destacar en primer término, su aspecto físico, notablemente obesa, y aniñada, peinada con diadema”.

Como ya nos advirtieran Verdugo et al. (2002) su sexualidad puede ser incluso negada, como observamos en la sentencia 92/2015 de 16 de febrero de la Audiencia Provincial de Málaga en la que se afirma "la incompatibilidad que tienen estos estados patológicos con un consentimiento verdadero y libre basado en el conocimiento de la transcendencia y significado del acto”.

Por último, cabe mencionar que en el 76,8 \% de las sentencias la terminología utilizada no es discapacidad intelectual, empleándose términos obsoletos como retraso mental, deficiencia mental o deficiencia psíquica. Esta confusión terminológica puede dar lugar a que la jurisprudencia equipare la DID con un trastorno mental, con las implicaciones que ello pudiera tener en la consideración del testimonio de la víctima.

\section{Discusión}

El presente trabajo pretendía analizar las posibles barreras que nuestro sistema judicial pudiera presentar a la hora de garantizar un adecuado acceso a la justicia a aquellas personas con DID que han sido víctimas de abusos y agresiones sexuales. Además, y dada la escasez de literatura sobre la comisión de este tipo de delitos en personas con DID, el estudio presentado analiza las características de las víctimas con DID y también las de quienes cometen el abuso o agresión, aportando información adicional sobre el proceso de denuncia.

De las 56 sentencias analizadas se desprende que las mujeres con DID y mayores necesidades de apoyo son víctimas especialmente vulnerables frente a este tipo de delitos. Si bien hemos de tener en cuenta que los datos referidos a quien interpone la denuncia pueden deberse a que un elevado porcentaje de víctimas estaban tuteladas, situación en la que es el representante legal quien interpone aquella, el escaso porcentaje de denuncias interpuestas por las víctimas debiera llevarnos a considerar la posibilidad de que no se les estén facilitando los apoyos adecuados tanto para reconocer este tipo de ataques como para denunciarlos.

Con respecto al perfil de quien comete la agresión, se caracteriza por pertenecer al círculo social cercano de la víctima en la mayor parte de los casos. Este dato nos lleva a enfatizar aún más la necesidad de dotar de herramientas a las personas con DID para identificar y revelar ${ }^{1}$ situaciones que pudieran atentar contra su integridad personal, en tanto que en el entorno próximo puede ser aún más difícil reconocer una situación de abuso y más complejo tramitar una denuncia si la persona depende de las personas de este contexto para hacerlo. Iniciativas como la web de información y apoyo http://nomasabuso.com/ o el Servicio de Protección de las Personas con

1 Recomendamos a los interesados la lectura del Manual de procedimiento para la atención de la Policía Local a las personas con discapacidad intelectual elaborado en 2017 por Plena inclusión España y la Unión Nacional de Jefes y Directivos de la Policía Local (UNIJEPOL) y que incluye material en lectura fácil sobre cómo denunciar https://www.plenainclusion.org/sites/default/files/manual-policialocal-15-06-17.pdf. 
Discapacidad (PROTEDIS) constituyen excelentes recursos en este sentido, pero no debemos olvidar que trabajar en la identificación de situaciones que atenten contra la integridad de la persona con DID no puede realizarse de manera puntual, sino que debemos garantizar que la persona, en todas las facetas de su vida, tenga oportunidades para conocer sus derechos, de modo que pueda denunciar cualquier atentando contra su dignidad.

En relación con las posibles barreras durante el proceso, nos gustaría destacar la falta de apoyos específicos a la que muchas víctimas con DID se enfrentan: la víctima declara en reiteradas ocasiones, especialmente cuando se juzga un delito de agresión sexual, sin ningún tipo de adaptación en más del $80 \%$ de los casos o apoyo profesional. Si bien el artículo 16 de la Convención de Naciones Unidas (2006), en vigor en España desde 2008, recoge la necesidad de realizar ajustes de procedimiento entre los que se encuentra la prueba preconstituida, esta fue utilizada con un $37,2 \%$ de las víctimas de abuso y tan solo con cuatro de las 24 víctimas de agresión sexual.

Como señalan Nixon et al. (2017) la carencia de apoyos durante el proceso incrementa el riesgo de revictimización y nos lleva a subrayar la necesidad de llevar a cabo intervenciones no solo de carácter primario que eviten el riesgo de las víctimas con DID ante la violencia sexual (véase en este sentido el protocolo de prevención de abuso de la Fundación A LA PAR elaborado por Recio et al., 2013), sino también, desgraciadamente, de carácter terciario, de modo que se garantice un adecuado acceso a la justicia. Estas intervenciones se recogen no solo en la Convención de Naciones Unidas a través del empleo de ajustes de procedimiento y la capacitación de los funcionarios encargados de velar y hacer cumplir la ley, sino en el más reciente Estatuto de la Víctima que, como expone con detalle De Araoz (2018), recoge en su artículo 26 medidas extraordinarias para víctimas necesitadas de especial protección.

No obstante, el empleo de estos ajustes de procedimiento parece depender más del trabajo realizado por el tercer sector que del llevado a cabo por nuestro sistema jurídico. Cabe destacar aquí la ya mencionada figura del facilitador en la investigación policial y judicial propuesta por la UAVDI de la Fundación A LA PAR (Recio et al., 2012). Destaca también el trabajo realizado por Silva, Manzanero y Contreras (2018) junto con el Ministerio de Interior para desarrollar un protocolo de evaluación de capacidades para testificar (CAPALIST) que facilite el derecho a la participación en el proceso, con las garantías necesarias, a aquellas víctimas necesitadas de mayor protección por su mayor vulnerabilidad, como personas con DID o menores de edad.

Quisiéramos mencionar también la necesidad de implicar a los actores jurídicos en un mayor conocimiento de las discapacidades del desarrollo, dado que los estereotipos con respecto a estas pueden agravar las consecuencias del abuso en la víctima. Observábamos que solo en el 30\% de los casos se valora el daño psicológico en la víctima. Incluso en un caso se decide no hacerlo por argumentos que atentan claramente contra la dignidad de la víctima y, por tanto, contra el articulado de la Convención y todos los tratados de derechos humanos que protegen a aquella. La realidad pone de manifiesto que las personas con DID experimentan las consecuencias psicológicas del abuso, y si bien pueden presentar sintomatología similar a la observada en la población general (Soylu, Alpaslan, Ayaz, Esenyel y Oruç, 2013), este tipo de situaciones 
exacerban o precipitan en este colectivo problemas de conducta (especialmente conductas autolesivas) (Gil-Llario, Morell-Mengual, Díaz-Rodríguez y Ballester-Arnal, 2018; Soylu et al., 2013). Es, además, más probable que experimenten reiterados episodios de victimización (Fisher et al., 2016; Nixon et al., 2017). Es urgente, por tanto, analizar hasta qué punto se trabaja posteriormente con la persona con DID en la elaboración de lo sucedido y en el desarrollo de estrategias y habilidades de prevención de delitos similares en el futuro (Fisher et al., 2016), pues la investigación hasta el momento existente pone de manifiesto que las personas con DID no suelen recibir el apoyo psicológico que pudieran necesitar (Gutiérrez-Bermejo, 2017; Peckham, Howlett y Corbett, 2007).

Si bien este estudio ha permitido arrojar algo más de información sobre una realidad prácticamente invisible, adolece de algunas limitaciones. Una de ellas hace referencia a los términos de búsqueda empleados. Si bien se decidió utilizar el término "discapacidad" al verse limitada la búsqueda a los últimos cuatro años, vemos que la terminología para referirse a esta condición en el ámbito jurídico está obsoleta, lo que pudiera haber limitado el acceso a determinadas sentencias en las que se utilizara solo el término de "retraso mental" o "deficiencia" sin aludir en ningún momento al concepto de discapacidad. Por otro lado, los cambios legislativos y de procedimiento como los introducidos por la Ley del Estatuto de la víctima del delito o el Convenio de Estambul han limitado el número de sentencias objeto de análisis a 56. Por otra parte, nos encontramos ante un estudio meramente descriptivo, que pretende arrojar algo de luz sobre una realidad poco conocida. Futuros estudios debieran plantearse la posibilidad de comparar la realidad de las víctimas con DI con la de sus iguales sin discapacidad, así como analizar si se observa una tendencia ascendente en el empleo de ajustes de procedimiento. Por último, y si bien ante situaciones que pudieran generar duda las dos primeras autoras analizaron la información de las sentencias por separado, esto no se hizo en todos los casos dada la objetividad de la información analizada (e. g., grado de discapacidad, prueba de cargo, edad de la víctima o el agresor, etc.), lo que pudiera afectar a la fiabilidad de nuestros análisis.

A pesar de estas limitaciones, los datos arrojados ponen de manifiesto que las víctimas con DID se encuentran en una situación de vulnerabilidad debido a la falta de adaptación de los procesos judiciales a sus necesidades de apoyo, lo que deriva en un incumplimiento del artículo 13 de la Convención. La provisión de ajustes de procedimiento adecuados requiere que sigamos esforzándonos en conocer la realidad de las víctimas con DID. La investigación sigue siendo aún escasa, tanto en lo referido a la participación de este colectivo en el proceso judicial, como en lo relacionado con las consecuencias que experimentan. Este cometido ha de combinarse con un trabajo de sensibilización y formación especializada de los profesionales del ámbito jurídico, dadas las carencias que nuestro sistema judicial presenta a la hora de adaptar el proceso judicial a las personas con discapacidades del desarrollo. 
LAS PERSONAS CON DISCAPACIDAD INTELECTUAL COMO VÍCTIMAS DE DELITOS

CONTRA LA LIBERTAD SEXUAL: UNA REALIDAD INVISIBLE

ARÁNZAZU VELLAZ ZAMORANO, PATRICIA NAVAS MACHO E INÉS DE ARAOZ SÁNCHEZ-DOPICO

\section{Referencias bibliográficas}

BeAIL, N. (2002). Interrogative suggestibility, memory and intellectual disability. Journal of Applied Research in Intellectual Disabilities, 15(2), 129-137. https://doi.org/10.1046/ j.1468-3148.2002.00108.x

Benedet, J. y Grant, I. (2007). Hearing the sexual assault complaints of women with mental disabilities. McGill Law Journal, 52, 243-289.

Beristain, A. (1994). Nueva criminología desde el derecho penal y la victimología. Madrid: Tirant Lo Blanch.

Brown, D., Lewis, C., Stephens, E. y Lamb, M. (2017). Interviewers” approaches to questioning vulnerable child witnesses: The influences of developmental level versus intellectual disability status. Legal and Criminological Psychology, 22(2), 332-349. https://doi. org/10.1111/lcrp.12104

Byrne, G. (2017). Prevalence and psychological sequelae of sexual abuse among individuals with an intellectual disability: a review of the recent literature. Journal of Intellectual Disabilities, 22(3), 294-310. https://doi.org/10.1177/1744629517698844

Cereceda, J., González, J. L., Sánchez, F., Herrera, D., López, J. J., Martínez, F. ... GóMEz, M. A. (2017). Informe sobre delitos contra la libertad e indemnidad sexual en España. Madrid: Ministerio del Interior.

Cereceda, J., Rodríguez, M., López, J. J., Sánchez, F., Herrera, D., Martínez, F. ... GóMEz, M. A. (2018). Informe sobre delitos contra la libertad e indemnidad sexual en España. Madrid: Ministerio del Interior.

De Araoz, I. (2018). Acceso a la justicia: ajustes de procedimiento para personas con discapacidad intelectual y del desarrollo. Madrid: Plena inclusión España.

Fisher, M. H., Baird, J. V., Currey, A. D. y Hodapp, R. M. (2016). Victimisation and social vulnerability of adults with intellectual disability: a review of research extending beyond Wilson and Brewer. Australian Psychologist, 51(2), 114-127. https://doi.org/10.1111/ ap. 12180

Ford, R. M., Griffiths, S., Neulinger, K., Andrews, G., Shum, D. H. y Gray, P. H. (2017). Impaired prospective memory but intact episodic memory in intellectually average 7-to 9-year-olds born very preterm and/or very low birth weight. Child Neuropsychology, 23(8), 954-979. https://doi.org/10.1080/09297049.2016.1216091

Gil-Llario, M. D., Morell-Mengual, V., Ballester-Arnal, R. y Díaz-Rodríguez, I. (2018). The experience of sexuality in adults with intellectual disability. Journal of Intellectual Disability Research, 62(1), 72-80. https://doi.org/10.1111/jir.12455

Greenspan, S., Switzky, H. N. y Woods, G. W. (2011). Intelligence involves risk-awareness and intellectual disability involves risk-unawareness: implications of a theory of common sense. Journal of Intellectual and Developmental Disability, 36(4), 246-257. https://doi.org /10.3109/13668250.2011.626759

Greenspan, S. y Woods, G. W. (2014). Intellectual disability as a disorder of reasoning and judgement: the gradual move away from intelligence quotient-ceilings. Current Opinion in Psychiatry, 27(2), 110-116. https://doi.org/10.1097/yco.0000000000000037

Griego, A. W., Datzman, J. N., Estrada, S. M. y Middlebrook, S. S. (2019). Suggestibility and false memories in relation to intellectual disability and autism spectrum disorder: a meta-analytic review. Journal of Intellectual Disability Research, 63(12), 1464-1474. https:// doi.org/10.1111/jir.12668

Ediciones Universidad de Salamanca / CC BY-NC-ND

Siglo Cero, vol. 52 (1), 2021, enero-marzo, pp. 7-26 
Gutiérrez-Bermejo, B. (2017). Víctimas invisibles. Análisis de un caso de maltrato desde la perspectiva de la víctima con discapacidad intelectual. Siglo Cero, 48(1), 9-21. http://dx.doi. org/10.14201/scero2017481921

Helton, J. J., Gochez-Kerr, T. y Gruber, E. (2018). Sexual abuse of children with learning disabilities. Child Maltreatment, 23(2), 157-165. https://doi.org/10.1177/1077559517733814

Hershkowitz, I. (2018). NICHD-protocol investigations of individuals with intellectual disability: a descriptive analysis. Psychology, Public Policy and Law, 24(3), 393. https://doi. org/10.1037/law0000170

Kebbell, M. R. y Hatton, C. (1999). People with mental retardation as witnesses in court: a review. Mental Retardation, 37(3), 179-187.

Manzanero, A. y González, J. L. (2015). Modelo holístico de evaluación de la prueba testifical (HELPT). Papeles del Psicólogo, 36(2), 125-138.

Manzanero, A., Recio, M., Alemany, A. y Cendra, J. (2013). Atención a las víctimas con discapacidad intelectual. Madrid: Fundación Carmen Pardo-Valcarce.

Mevissen, L. y De Jongh, A. (2010). PTSD and its treatment in people with intellectual disabilities: a review of the literature. Clinical Psychology Review, 30(3), 308-316. https://doi. org/10.1016/j.cpr.2009.12.005

Naciones Unidas. (2006). Convention on the Rights of Persons with Disabilities. Recuperado de http://www.un.org/disabilities /convention/ conventionfull.shtml

Naciones Unidas. (2019). Observaciones finales sobre los informes periódicos segundo y tercero combinados de España. Recuperado de http://www.convenciondiscapacidad. es/2019/04/10/observaciones-finales-sobre-los-informes-periodicos-segundo-y-tercerocombinados-de-espana-del-comite-sobre-los-derechos-de-las-personas-con-discapacidad-9-de-abril-de-2019/

Navas, P., Gómez, L. E., Verdugo, M. Á. y Schalock, R. L. (2012). Derechos de las personas con discapacidad intelectual: implicaciones de la Convención de Naciones Unidas. Siglo Cero, 43, 7-28.

Nixon, M., Thomas, S. D., Daffern, M. y Ogloff, J. R. (2017). Estimating the risk of crime and victimisation in people with intellectual disability: a data-linkage study. Social Psychiatry and Psychiatric Epidemiology, 52(5), 617-626. https://doi.org/10.1007/s00127-0171371-3

Oficina del Alto Comisionado de las Naciones Unidas para los Derechos HuMANos. (2017) Informe sobre el Derecho de acceso a la justicia en virtud del artículo 13 de la Convención sobre los Derechos de las Personas con Discapacidad. Recuperado de https://documents-dds-ny.un.org/doc/UNDOC/GEN/G17/368/76/PDF/G1736876. pdf?OpenElement

Palomino, E., López-Frutos, J. M., Botella, J. y Sotillo, M. (2019). Impairment of cognitive memory inhibition in individuals with intellectual disability: a meta-analysis. Psicothema, 31(4), 384-392. https://doi.org/10.7334/psicothema2019.107

Peckham, N. G., Howlett, S. y Corbett, A. (2007). Evaluating a survivors group pilot for women with significant intellectual disabilities who have been sexually abused. Journal of Applied Research in Intellectual Disabilities, 20(4), 308-322. https://doi.org/10.1111/ j.1468-3148.2006.00347.x

Perske, R. (2008). False confessions from 53 persons with intellectual disabilities: the list keeps growing. Intellectual and Developmental Disabilities, 46(6), 468-479. https://doi. org/10.1352/2008.46:468-479 
Recio, M., Alemany, A. y Manzanero, A. L. (2012). La figura del facilitador en la investigación policial y judicial con víctimas con discapacidad intelectual. Siglo Cero, 43(3), 54-68.

Recio, M., Galindo, L., Cendra, J., Alemany, A., Villaró, G. y Martorell, A. (2013). Abuso y discapacidad intelectual: orientaciones para la prevención y la actuación. Madrid: Fundación Carmen Pardo-Valcarce.

Recover, T. y De Araoz, I. (2014). Las personas con discapacidad intelectual o del desarrollo ante el proceso penal. Madrid: FEAPS.

Silva, E. A., Manzanero, A. L. y Contreras, M. J. (2018). CAPALIST: Valoración de capacidades para testificar. Madrid: Dykinson.

Soylu, N., Alpaslan, A. H., Ayaz, M., Esenyel, S. y Oruç, M. (2013). Psychiatric disorders and characteristics of abuse in sexually abused children and adolescents with and without intellectual disabilities. Research in Developmental Disabilities, 34(12), 4334-4342. https:// doi.org/10.1016/j.ridd.2013.09.010

Spencer, N., Devereux, E., Wallace, A., Sundrum, R., Shenov, M., Bacchus, C. y Logan, S. (2005). Disabling conditions and registration for child abuse and neglect: a populationbased study. Pediatrics, 116, 609-613.

Subijana, I. J. y Echeburúa, E. (2018). Las menores víctimas de abuso sexual en el proceso judicial: el control de la victimización secundaria y las garantías jurídicas de los acusados. Anuario de Psicología Jurídica, 28(1), 22-27. https://doi.org/10.5093/apj2018a1

Verdugo, M. Á., Alcedo, M., Bermejo, B. y Aguado, A. L. (2002). El abuso sexual en personas con discapacidad intelectual. Psicothema, 14(Suplemento), 124-129.

Wissink, I. B., Van Vugt, E. S., Smits, I. A., Moonen, X. M. y Stams, G. J. J. (2017). Reports of sexual abuse of children in state care: a comparison between children with and without intellectual disability. Journal of Intellectual and Developmental Disability, 43(2), 152-163. https://doi.org/10.3109/13668250.2016.1269881

Withers, P. y Morris, J. (2012). Sexual exploitation of people with intellectual disabilities. En E. Emerson, C. Hatton, K. Dickson, R. Gone, A. Caine y J. Bromley (Eds.), Clinical Psychology and People with Intellectual Disabilities (pp. 273-292). Londres: John Wiley and Sons. 